\title{
Effets des biomasses des légumineuses ligneuses sur la croissance et le rendement du maïs en couloirs sur un ferralsol de Yangambi, RD Congo
}

\author{
Likoko Bambele Alexandre*1, Murefu Kashuva1, Likoko Abaya Georges ${ }^{1}$ Et Posho Ndola Boniface ${ }^{2}$ \\ 1-Institut National pour l'Etude et la Recherche Agronomiques (INERA), B.P. 2037, Kinshasa/Gombe, République \\ Démocratique du Congo (RD Congo), Centre de Recherche de Yangambi 2-Professeur à l'IFA Yangambi. \\ *Correspondant : LIKOKO BAMBELE, e-mail : bambelelikoko@yahoo.fr, Téléphone : (+243) 992414920
}

Original submitted in on $5^{\text {th }}$ June 2018. Published online at www.m.elewa.org on $30^{\text {th }}$ November 2018 https://dx.doi.org/10.4314/jab.v131i1.11

\begin{abstract}
RESUME
Une des solutions de lutter contre l'agriculture itinérante en préservant la réserve biosphérique de Yangambi est la culture en couloir aroforestier. Quatre traitements de légumineuses ligneuses ont été comparés pour leur pouvoir fertilisant sur le rendement du maïs pendant trois saisons culturales successives en bloc complet randomisé de cinq blocs et cinq traitements sur un ferralsol de la série $Y_{2}$ à Yangambi en vue de stabiliser durablement la culture Les productions de maïs ont significativement donné un rendement de $11,66 \%$ et $264 \%$ sous les haies de Leucaena leucocephala et de $29,60 \%$ et $226 \%$ sous celles de Cassia spectabilis par rapport au témoin avec $2658 \mathrm{~kg} / \mathrm{ha}$ et $1164 \mathrm{~kg} / \mathrm{ha}$ à la deuxième et troisième campagnes culturales, respectivement. Cependant, les arbustes bisannuels n'avaient de l'influence qu'à la première saison avec des rendements relatifs de 108,4\% sous Tephrosia vogelii et $113,2 \%$ sous Sesbania sesban par rapport au témoin qui a produit $2.514 \mathrm{~kg} / \mathrm{ha}$, contre $88,5 \%$ sous L.leucocephala et $79,1 \%$ sous C..spectabilis. Les arbustes à usage multiple le local C.spectabilis et la légumineuse exotique L.leucocephala valorisent les courtes jachères herbeuses et évitent l'itinérance agricole en fertilisant le même sol pendant plusieurs années en matière organique, azote et éléments minéraux, et peuvent être étendue en milieu paysan. Par contre, T.vogelii et S. sesban dépérissant après le premier recepage exigent la replantation chaque année et une main d'œuvre supplémentaire.
\end{abstract}

Mots clés : Culture en couloire, légumineuses ligneuses, biomasses, rendement du maïs et Yangambi

\section{SUMMARY}

Ligneous legumes biomass effect on the growth and yield of maize in Yangambi

One of alleviations to control shifting cultivation in conserving Yangambi biosphere reserve is the agroforetry alley cropping. Four woody legumes were evaluated for their biomass potential fertility on maize production during three consecutive cultural seasons in randomised complet block designe of five blocks and five treatments on a ferralsol $\mathrm{Y}_{2}$-soil series in Yangambi to stabilise sustainably the cropping. Maize has significantly yielded $11.66 \%$ and $246 \%$ under Leucaena leucocephala and $29.60 \%$ and $226 \%$ under Cassia spectabilis hedgerows as compared to the control with 2658 and $1164 \mathrm{~kg} / \mathrm{ha}$ in the second and third seasons, respectively. However, the influence of biannual species on relative maize production was 
observed only in the first season with $108.4 \%$ under Tephrosia vogelii and $113.2 \%$ under Sesbania sesban facing the control with $2514 \mathrm{~kg} / \mathrm{ha}$, against $88.5 \%$ under L.leucocephala and $79.1 \%$ under C.spectabilis. The polyvalent shrubs the indigenous C.spectabilis and the exotic legume L.leucocephala valorise shorterned herbaceous fallow and avoid agricutural shifting in fertilizing the same soil with organic matter, nitrogen and mineral elements during several years ; and could be extended in farming system. On the other hand, T.vogelii and S.sesban perishing after the first slashing, need every year replantation and supplemantary manpower.

Keywords: Alley cropping, woody legumes, biomass, maize yield and Yangambi.

\section{INTRODUCTION}

La perte de fertilité des sols kaolinitiques de Yangambi a conduit l'agriculteur à continuer son système traditionnel d'agriculture itinérante sur abattis brûlis pratiquant des cultures durant trois ans et mettant le sol en jachère pendant huit à quatorze ans afin de régénérer la fertilité des terres fatiguées (Alongo et al., 2013; Jurion et Henri, 1967). Cette période de jachère est souvent écourtée à quatre à six ans par la population de 76.000 habitants à croissance démographique de $2,9 \%$ par an dans la région de la réserve écologique de Yangambi qui couvre 440.000 hectares, soit une densité de 132 habitants par $\mathrm{Km}^{2}$ de la zone de transition biosphérique (Alongo et al., 20013 ; INERA,2010). Pourtant, les arbustes polyvalents utilisés en cultures extensives en couloir pourraient jouer un rôle satisfaisant d'une jachère améliorée et plus courte. Ce système dénommé « Agroforesterie » a donc l'avantage de produire le paillage et le fertilisant engrais vert pour régénérer rapidement la fertilité du sol en allongeant la période culturale à cinq ans jusqu'à dix ans, et de stabiliser la production vivrière (FAO, 2000 ; Kang et al., 1995). En effet, les biomasses de ces arbustes utilisées en agroforesterie fournissent dans le sol trois fois plus d'éléments minéraux ( $\mathrm{N}, \mathrm{P}, \mathrm{K}, \mathrm{C}$ organique, $\mathrm{Ca}$ et $\mathrm{Mg}$ ) par hectare par an pendant plus de cinq ans que la jachère naturelle (FAO, 2000 ; Kang et al., 1995). Pour le Leucaena leucocephala, $70 \%$ d'azote proviendrait de la fixation symbiotique de l'azote libre de l'air par un rhizobium hautement spécifique, augmentant ainsi la quantité d'azote combiné dans l'alfisol estimé à $40 \mathrm{~kg} / \mathrm{ha} / \mathrm{an}$ en couloir de maïs (Van Laure et al., 2000 ; Sanginga et al., 1995 ; Tian et al., 1994). Sur des ferralsols, le $L$. leucocephala en couloire a élevé la production de bananier de 7.2 à 16 tha (Mate, 2003). Le C. spectabilis a été apprécié sur des ultisols acides fournissant des biomasses à minéraux majeurs comparables à $L$. leucocephala (Kang et al., 1997; Kang et al., 1995]. A Yangambi, il est fréquent (10\%) et abondant $(1,68$ pieds $/ \mathrm{m}^{2}$ ) dans des jachères herbacées (Likoko et Tentula, 2003). Le système agro-forestier par $L$. leucocephala et $C$. spectabilis s'est aussi adapté aux pâturages en produisant une alimentation animale de haute qualité. Le $L$. leucocephala seul produit des jeunes gousses et graines pour l'alimentation humaine, de bois d'œuvre et de la pâte à papier (FAO, 2000). II sert aussi d'arbre d'ombrage, couverture, jachère, antiérosif et fourrager. Sesbania sesban et le Tephrosia vogelii sont des arbustes utilisés pour la jachère de courte durée, ayant des croissances végétatives très rapides et fournissant beaucoup plus de biomasses foliaires que ligneuses qui contiennent des teneurs en éléments minéraux mobiles $(\mathrm{N}, \mathrm{Ca}$ et $\mathrm{Na}$ ) plus que le L.leucocephala et C.spectabilis (Kang et al., 1997; Kang et al., 1995). Ceci entraînerait une faible utilisation d'engrais chimiques protégeant ainsi l'environnement contre les pollutions (Kang et al., 1995). Aussi, la matière organique et l'ombrage des mulches de ces arbustes stimulent l'activité des vers de terre et des microarthropodes du sol qui, par leurs déjections, augmentent la fertilité minérale et organique du sol à un point correspondant à celle d'une forêt secondaire (Tian et al., 1994). Les mulches de $L$. leucocephala et $S$. sesban luttent également contre l'érosion et les mauvaises herbes, réduisent le ruissellement d'eau de pluie au sol en améliorant la rétention d'eau à la surface du sol, l'infiltration d'eau dans le sol et la densité 
du sol (Kang et al., 1997 ; Tian et al., 1994). Le T. vogelii est aussi utilisé pour la pêche pour son action convulsive et paralysante (Letouzey et White, 1978). Bien que bien adaptées sur les alfisols et les ultisols, les légumineuses ligneuses polyvalentes $L$. leucocephala, $T$. vogelii et $S$. sesban nécessitent d'être expérimentées sur le

\section{MATERIELS ET METHODES}

Milieu d'étude: Cette étude a été conduite à Yangambi $\left(0^{\circ} 49^{\prime} \mathrm{N}\right.$ et $24^{\circ} 29^{\prime} \mathrm{E}$, à 470 mètres d'altitude au-dessus du niveau de la mer), à environ $100 \mathrm{~km}$ à l'Ouest de la ville de Kisangani en RD Congo. Le site expérimental appartient au climat Af selon Köppen, avec des moyennes annuelles de $1.811 \mathrm{~mm}$ des pluies, $24,9^{\circ} \mathrm{C}$ de température moyenne, 2.024 heures d'insolation et $1.186 \mathrm{~mm}$ d'évapotranspiration potentielle (Likoko et al., 2014). Le sol du site est un ferralsol de la série $Y_{2}$ Yakonde du plateau Isalowe. Ses caractéristiques physicochimiques sous forêt primaire sont un $\mathrm{pH}_{\mathrm{H} 2 \mathrm{O}}$ de 4,6, une capacité d'échange cationique (CEC, à l'acétate d'ammonium pH7) de 3,82 méq $/ 100 \mathrm{~g}$ de sol, une somme des bases échangeables ferralsol de Yangambi. L'objectif de cette étude est donc de comparer à l'espèce locale $C$. spectabilis pour leur pouvoir d'amélioration de la fertilité du sol, exprimé en termes de rendement stabilisé de maïs cultivé en couloir de haies de ces quatre arbustes.

de 0,22 méq/100g de sol, une texture sablo-argileuse et une structure granuleuse fine à polyédrique subangulaire d'activité biologique et enracinement généralement intenses de 0 à $30 \mathrm{~cm}$ de profondeur (Alongo et al., 2013 ; Kombele, 2004).

Matériels: Quatre légumineuses ligneuses dont $L$. leucocephala $K 8, T$. vogelii et $S$. sesban provenues de I'Institut International d'Agriculture Tropicale (IITA) et $C$. spectabilis, légumineuse locale, introduites dans la collection agro-forestière de l'INERA en 1989, ont été évaluées. Selon Letouzey et White (1978), les caractéristiques botaniques de ces légumineuses sont présentées au tableau 1.

Tableau1. Caractéristiques botaniques des arbustes utilisés dans la culture de maïs.

\begin{tabular}{|l|l|l|}
\hline Espèces & Famille botanique & Caractéristiques botaniques \\
\hline $\begin{array}{l}\text { Leucaena } \\
\text { leucocephala }\end{array}$ & Mimosaceae & $\begin{array}{l}\text { Espèce ligneuse vivace sans épine à feuilles bipennées, } \\
\text { acclimaté en savane de sols neutres ou basiques et peut } \\
\text { atteindre 10m de haut. }\end{array}$ \\
\hline Cassia spectabilis & Caesalpiniaceae & $\begin{array}{l}\text { Espèce spontanée en cuvette congolaise, s'adapte dans toute } \\
\text { la RD Congo jusqu'à 1700m d'altitude avec un excellent } \\
\text { pouvoir de rejeter des souches et une formation rapide de l'état } \\
\text { massif végétal. Il est utilisé pour l'ornement et le reboisement } \\
\text { pour de bois de chauffage, coupe-feu, brise-vent et perches. }\end{array}$ \\
\hline Tephrosia vogelii & Fabaceae & $\begin{array}{l}\text { Arbuste robuste ramifié de 1 à 4m de haut, de tige densément } \\
\text { veloutée à poils courts et longs étalés mélangés. Les feuilles } \\
\text { sont acuminées de 1à 2cm de long et caduques }\end{array}$ \\
\hline Sesbania sesban & Fabaceae & $\begin{array}{l}\text { Plante ligneuse généralement annuelle ou bisannuelle de 1 à } \\
6 \mathrm{~m} \text { de haut. Ses feuilles à stipules lancéolées ou de forme plus } \\
\text { étroite de 4 à 8mm de long sont caduques parfois } \\
\text { subpersistantes et cantortées }\end{array}$ \\
\hline
\end{tabular}

La variété de maïs Kasaï 1 fixée par l'INERA (variété à grains de couleur blanche, à cycle végétatif de 75 jours et produisant le rendement de $2,5 \mathrm{t} / \mathrm{ha}$ en station) a été utilisée comme matériel végétal. Le précédent cultural était une jachère de 6 ans colonisée par les espèces Musanga cecropiodes, Mariscus glabeliformis, Comelina diffusa, Aneliena ambrosum, Panicum repens et Mimosa invisa.
Méthodes: Le dispositif expérimental utilisé était le bloc complet randomisé à 5 blocs et 5 traitements pour quatre répétitions dans le temps. Les traitements appliqués étaient constitués des haies des arbustes de 4 légumineuses (L. leucocephala K8, T. vogelii, S. sesban et $C$. spectabilis) et le témoin, le traitement sans arbuste. Le maïs était semé dans les couloirs aux écartements de $0,80 \mathrm{~m} \times 0,50 \mathrm{~m}$, à raison de deux 
graines par poquet. Trois saisons culturales successives (2007-2010) ont été organisées. Dans l'essai préliminaire d'adaptation, pour accélérer le taux de germination, les graines des arbustes étaient traitées à l'eau chaude pendant une minute et semées une fois par mois pendant 11 mois, aux écartements de $4 \mathrm{~m} \times 0,5 \mathrm{~m}$ en raison de deux graines par poquet. Pour chaque saison culturale du maïs, deux tailles des arbustes étaient faites à $0,10 \mathrm{~m}$ du sol : deux semaines avant et quatre semaines après semis de maïs. La biomasse foliaire (t/ha) de chaque traitement d'arbuste récolté par saison culturale préalablement pesée était épandue dans la parcelle correspondante. Les échantillons de biomasses foliaires de chaque espèce par saison culturale, ont été séchés à l'air libre, moulus au pilon et mortier et ensuite soumis à la digestion humide avec la solution de $\mathrm{HClO}_{4}-\mathrm{HNO}_{3}$. La solution filtrée au moyen de papier filtre a été recueillie pour analyser les éléments minéraux au laboratoire de I'INERA Mulungu. Le potassium, le calcium et le magnésium ont été dosés au spectrophotomètre d'absorption atomique. Le phosphore était dosé par la méthode de Vana-Molybdate (bleu de moly) à l'aide d'un colorimètre (Landon, 1991). Les résultats de biomasses montrés au tableau 2 sont les moyennes de trois saisons culturales. Les échantillons composites de sols prélevés dans $30 \mathrm{~cm}$ de profondeur dans chaque

\section{RESULTATS ET DISCUSSIONS}

Comportement des légumineuses ligneuses à Yangambi: Le comportement des légumineuses ligneuses introduites à Yangambi est présenté au tableau 2. Plus de $90 \%$ de graines d'arbustes pérennes $L$. leucocephala et $C$. spectabilis ont germé dans les cinq jours après le semis. Leur période de dormance avait atteint sept mois pour $C$. spectabilis et 11 mois pour $L$. leucocephala avec $10 \%$ de germination. Plus de $70-80 \%$ de graines des arbustes bisannuels $T$. vogelii et $S$. sesban ont germé dans un mois et qu'à quatre mois le taux de germination était réduit à $10 \%$. Pendant que le C. spectabilis n'a pas nodulé, le $T$. vogelii et le $S$. sesban ont nodulé plus abondamment que le $L$. leucocephala. Ces espèces bisannuelles étaient plus précoces que les pérennes. A 10 mois, le $S$. sesban avait atteint une hauteur de $456 \mathrm{~cm}$ et un diamètre légèrement supérieur $(4,9 \mathrm{~cm})$ à $T$. vogelii $(4,6 \mathrm{~cm})$. Malgré sa plus petite hauteur de $176 \mathrm{~cm}$, cette dernière, a donné la plus grande biomasse à la première saison parcelle d'un traitement étaient séchés à l'air libre puis tamisé sur maille de $2 \mathrm{~mm}$. La terre fine $(<2 \mathrm{~mm})$ était utilisée pour analyser seulement le pH du sol mesuré à l'aide d'une électrode de verre combinée trempant dans une suspension de sol (rapport eau/sol : 2,5) au moyen d'un pH-mètre (Type Electronic model 6054 $\left.N^{\circ} 16 / 2464\right)$ (Landon, 1991). Pour le maïs, les paramètres d'évaluation ont porté sur la hauteur $(\mathrm{cm})$, le diamètre au collet $(\mathrm{cm})$ des plants à la floraison mâle et le rendement du maïs Kasal̈ 1 (kg/ha). Pour les légumineuses utilisées comme haies, les nodules (10 pieds par traitement de chaque espèce d'arbustes) ont été comptées, converties en pourcentage et classifiées en très abondant $(>70 \%)$, abondant $(50-69 \%)$ et moins abondant $(<50 \%)$. La précocité des arbustes était évaluée par la croissance en hauteur et en diamètre au collet à l'âge de 10 mois après semis. Leur aptitude en hauteur était déterminée par la reprise végétative après recépages et élagages. Le taux de germination, les temps en mois d'atteindre la floraison et la période de floraison en cours de l'année ont été aussi observés. Les analyses statistiques ont consisté dans la détermination de la moyenne, de l'écart-type, du coefficient de variation et de l'analyse de la variance (ANOVA). Le test de la plus petite différence (LSD 5\%) a été appliqué pour différencier les moyennes des paramètres étudiés.

culturale. II est important de signaler que ces deux espèces ( $S$. sesban et $T$. vogeli) n'étaient pas aptes à reprendre la végétation après les deux tailles de la première saison culturale, alors que sur des alfisols acides de savane, ils reprenaient la végétation à plusieurs recepages. Ils avaient atteint la floraison à 6-7 mois et pouvaient fleurir en toute période de l'année. Les espèces pérennes ( $L$. leucocephala et $C$. spectabilis), avaient montré une grande aptitude à la reprise végétative continuelle après des tailles et élagages. L'espèce $L$. leucocephala avait la plus grande taille $(275 \mathrm{~cm})$ par rapport à $C$. spectabilis $(208 \mathrm{~cm})$, et avait également fleuri plus tôt (à 13 mois contre 29 mois pour $C$. spectabilis). Ils produisent des biomasses ligneuses de l'ordre de 15 tha utilisables à Yangambi comme bois de chauffage et de petites constructions zootechniques (Alongo et al., 2014, INERA, 2010 ; Kang et al., 1997). 
Likoko et al., J. Appl. Biosci. 2018 Effets des biomasses des légumineuses ligneuses sur la croissance et le rendement du maïs en couloirs sur un ferralsol de Yangambi, RD Congo

Tableau 2 : Comportements d'adaptation de quatre légumineuses vertes introduites en 1989 sur le ferralsol $Y_{2}$ à Yangambi.

\begin{tabular}{|c|c|c|c|c|c|c|c|c|c|c|c|c|c|}
\hline \multirow{4}{*}{$\begin{array}{l}\text { Engrais verts } \\
\text { (légumineuses } \\
\text { ligneuses) }\end{array}$} & \multicolumn{5}{|c|}{ Germination (\%) } & \multirow{4}{*}{$\begin{array}{l}\frac{.}{0} \\
\frac{0}{\bar{t}} \\
\frac{7}{2} \\
\frac{0}{2}\end{array}$} & \multirow{2}{*}{\multicolumn{2}{|c|}{$\begin{array}{c}\text { Précocité à } 10 \\
\text { mois }\end{array}$}} & \multirow{2}{*}{\multicolumn{2}{|c|}{$\begin{array}{l}\text { Aptitude à la } \\
\text { taille }\end{array}$}} & \multirow[t]{4}{*}{$\begin{array}{c}\text { Décomposition } \\
\text { de biomasse }\end{array}$} & \multirow{4}{*}{$\begin{array}{l}\text { Floraison } \\
\text { (mois, } \\
\text { après } \\
\text { semis) }\end{array}$} & \multirow[t]{4}{*}{$\begin{array}{l}\text { Période de } \\
\text { floraison }\end{array}$} \\
\hline & & & lois & & & & & & & & & & \\
\hline & \multicolumn{2}{|c|}{1} & \multirow[t]{2}{*}{4} & \multirow[t]{2}{*}{7} & \multirow[t]{2}{*}{11} & & \multirow{2}{*}{ 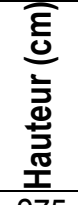 } & \multirow{2}{*}{ 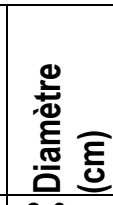 } & \multirow{2}{*}{ 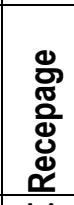 } & \multirow[b]{2}{*}{ 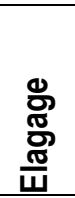 } & & & \\
\hline & $\begin{array}{l}\leq \\
5 \text { jrs }\end{array}$ & $\begin{array}{l}6-30 \\
\text { jrs }\end{array}$ & & & & & & & & & & & \\
\hline \begin{tabular}{|l|} 
Leucaena \\
leucocephala
\end{tabular} & 92 & - & - & - & 10 & * & 275 & 2.0 & $\overline{\Delta \Delta}$ & $\Delta \Delta$ & $\begin{array}{l}\text { Peu rapide } \\
\text { (2-3 mois) }\end{array}$ & 13 & Avril-octobre \\
\hline Cassia spectabilis & 90 & - & - & 10 & - & - & 208 & 2.0 & $\Delta \Delta$ & $\Delta \Delta$ & $\begin{array}{l}\text { Moins rapide } \\
\text { (2-4 mois) }\end{array}$ & 29 & Juillet-février \\
\hline Tephrosia vogelii & - & 70 & 10 & - & - & ** & 176 & 4.6 & - & $\Delta$ & $\begin{array}{l}\text { Rapide (3-6 } \\
\text { semaines) }\end{array}$ & 7 & Tout l'an \\
\hline Sesbania sesban & - & 80 & 10 & - & - & ** & 456 & 4.9 & - & - & $\begin{array}{l}\text { Rapide (3-6 } \\
\text { semaines) }\end{array}$ & 6 & Tout l'an \\
\hline
\end{tabular}

Légende : *=abondant $50-69 \%,{ }^{* *}=$ très abondant $\geq 70 \%, \Delta=$ apte à reprendre après taille, $\Delta \Delta=$ très apte à reprendre la taille, -=néant, jrs=jours. 
Likoko et al., J. Appl. Biosci. 2018 Effets des biomasses des légumineuses ligneuses sur la croissance et le rendement du maïs en couloirs sur un ferralsol de Yangambi, RD Congo

L'effet des biomasses des légumineuses vertes sur la croissance et le rendement du maïs en première saison : Les résultats relatifs aux moyennes des diamètres au collet, hauteurs des plants à la floraison mâle et rendements du maïs en couloir de haies de quatre légumineuses ligneuses pendant trois saisons culturales successives sont présentés aux figures $1 \mathrm{a}, 1 \mathrm{~b}, 1 \mathrm{c}$ et $1 \mathrm{~d}$.

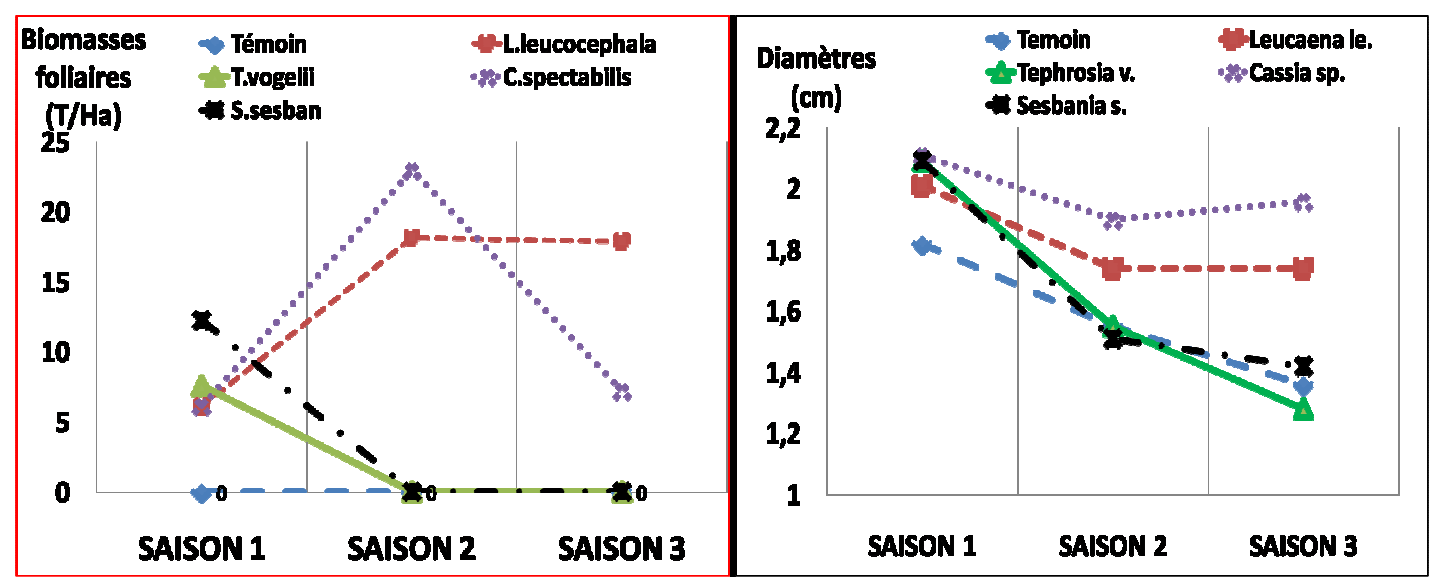

Figure 1a, Evolution des biomassesde Lleurcocephola, C.spectobilits, T, Twogelii et $S$.sesbon en trois saisons culturales sucessivesà Yangambi.

Figure $1 \mathrm{~b}$. Evolution en diamètre au collet du mais sous L.Jeurocephala, C.spectabilis, T.vogelit et S.seshon en trois saisons culturales successives à Yangambi

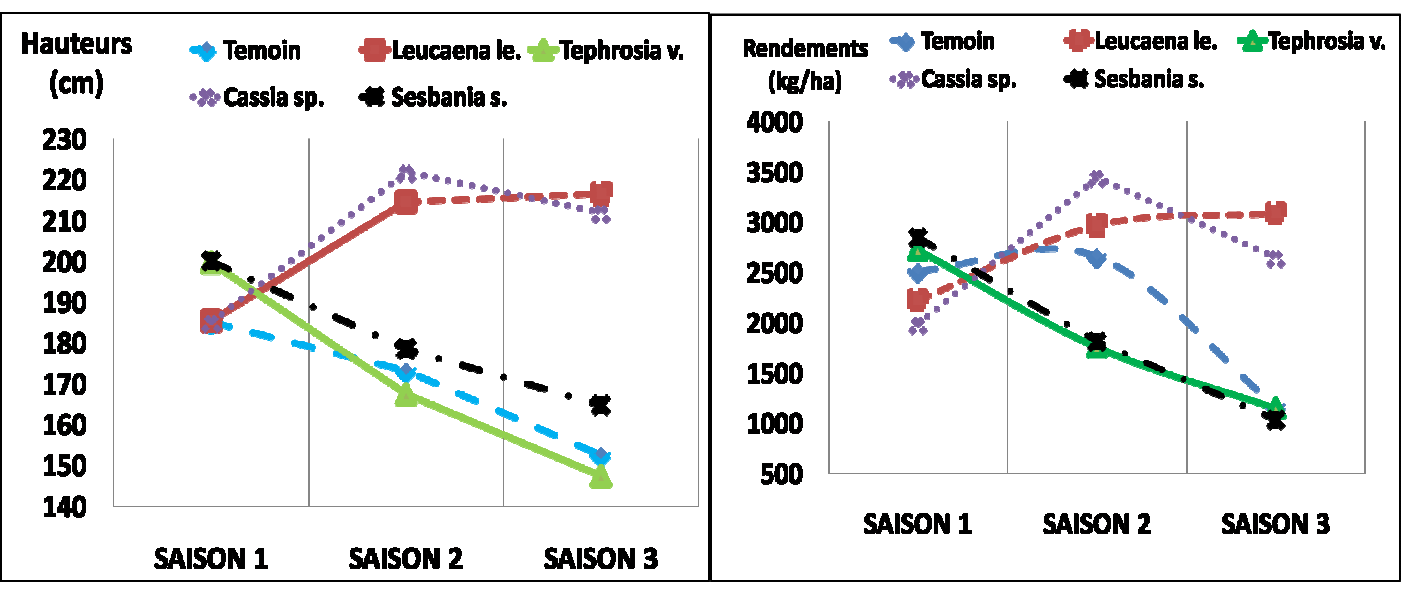

Figure 1c. Evolutionen hauteur du mais sous Lleucocephoha, C.spectabilis, Tvogelijet S.sebon en trois saisons culturales successives à Yangambi.

Figure 1d. Evolution de rendements du mails sous Llevecocephada, Cspectabilits, Trogethiet S.sesban en trois saisons culturales sutcessives à Yangantbi. 
Tableau 3. Moyennes de trois saisons culturales successives du diamètre au collet, de la hauteur et du rendement du maïs Kasaï1 en fonctions de différents traitements d'engrais verts à Yangambi, RDC.

\begin{tabular}{|c|c|c|c|c|c|c|c|c|c|}
\hline \multirow[b]{3}{*}{ Traitements } & \multirow{3}{*}{$\begin{array}{l}\text { Diometre } \\
(\mathrm{cm})\end{array}$} & \multicolumn{2}{|l|}{ Maís } & \multicolumn{5}{|c|}{$\begin{array}{c}\text { Biomasses foliaires dengrais } \\
\text { verts }\end{array}$} & \multirow{3}{*}{$\begin{array}{l}\text { pHeau } \\
\text { du sol }\end{array}$} \\
\hline & & Hanter- & Dandamant & \multirow[b]{2}{*}{ t/ho } & \multicolumn{4}{|c|}{$\begin{array}{l}\text { Eléments Minéraux } \\
\text { Majeurs (kg/ha) }\end{array}$} & \\
\hline & & $(\mathrm{cm})$ & (kg/ha) & & $\boldsymbol{P}$ & $\mathbf{K}$ & $\mathbf{C a}$ & $\mathbf{M g}$ & \\
\hline Témoln & $1,58+0,18 c$ & $170,23 \pm 23,29 b$ & $2110,67 \pm 672,12$ & - & - & & & & 4,88 \\
\hline $\begin{array}{l}\text { Leucaena } \\
\text { leucocephala. }\end{array}$ & $1.83 \pm 0,14 b$ & $205,54 \pm 14,240$ & $2759,20 \pm 384,41$ & $\begin{array}{c}42,110 \pm \\
6,88\end{array}$ & 13,9 & 73,7 & 235,3 & 18,6 & 5,71 \\
\hline $\begin{array}{l}\text { Tephrosla } \\
\text { vogellI }\end{array}$ & $1,64 \pm 0,33 c$ & $171,53 \pm 21,45 b$ & $1986,27 \pm 538,88$ & $7,59 c$ & & & & & 6,22 \\
\hline $\begin{array}{l}\text { Cassla } \\
\text { spectabllis }\end{array}$ & $1,99 \pm 0,09 a$ & $206,27 \pm 15,56$ & $2694,53 \pm 535,84$ & $\begin{array}{c}36,38 b \pm \\
9,42\end{array}$ & 17,7 & 88,9 & 172,2 & 18,9 & 5,53 \\
\hline $\begin{array}{l}\text { Sesbanla } \\
\text { esban }\end{array}$ & $1,67 \pm 0,30 c$ & $181,14 \pm 14,49 a$ & $2092,13 \pm 541,83$ & $12,13 c$ & & & & & 5,71 \\
\hline Moyennes & $1,74 \pm 0,10$ & $186,94 \pm 18,94$ & $2328,58 \pm 161,18$ & $24,55 \pm 6.79$ & & & & & \\
\hline$c v(\%)$ & 5,71 & 10,44 & 24,1 & $\mathbf{9 0 , 9 5}$ & & & & & \\
\hline LSD 5\%) & 0,13 & 25,36 & NS & 5.54 & & & & & \\
\hline
\end{tabular}

Légende : *=significatif, ${ }^{* *}=$ hautement significatif et ns=non significatif. Les moyennes suivies des mêmes lettres ne sont pas significativement différentes au seuil $a=0,5$.

En première saison (figure 1a), les biomasses des légumineuses bisannuelles (T.vogelii et S.sesban) étaient plus élevées respectivement 7,59 tha et 4,15 tha que celles des légumineuses pérennes, probablement suite à leur précocité en hauteur avec beaucoup de petites branches susceptibles de porter une grande végétation. Aussi, leur abondance nodulaire accélérerait la fixation azotée favorable à la formation d'une grande densité foliaire totale. A cause de leur dépérissement, faudrait-il renouveler la plantation de ces arbustes bisannuels à chaque saison culturale. Quant à la croissance du maïs, la figure $1 \mathrm{~b}$ montre que le témoin avait un diamètre significativement inférieur $(1,82 \mathrm{~cm})$ à ceux de tous les autres traitements, lesquels étaient comparables. Toutefois, le diamètre du maïs traité au $C$. spectabilis était relativement le plus élevé $(2,11$ $\mathrm{cm})$. En absence de T.vogelii et S.sesban dépéris, les diamètres du maïs avaient respectivement diminué de $2,08 \mathrm{~cm}$ à $1,30 \mathrm{~cm}$ et $1,40 \mathrm{~cm}$ et étaient comparables à celui du témoin $1,40 \mathrm{~cm}$ en troisième saison culturale. Les moyennes de hauteur et de rendement du maïs n'ont pas montré de différences significatives à la première saison (figures $1 \mathrm{c}$ et $1 \mathrm{~d}$, saison 1). Malgré cette tendance, la hauteur $(\sim 200 \mathrm{~cm})$ et le rendement du maïs sous $T$. vogelii $(2.726$ $\mathrm{kg} / \mathrm{ha}$ ) et $S$. sesban $(2.846 \mathrm{~kg} / \mathrm{ha})$, arbustes bisannuels, sont légèrement supérieurs à ceux des arbustes pérennes ( $L$. leucocephala et $C$. spectabilis) et du témoin, soit une hauteur d'environ $185 \mathrm{~cm}$ pour ces trois traitements et un rendement de l'ordre $2.224,1.988$ et $2.514 \mathrm{~kg} / \mathrm{ha}$ pour L. leucocephala et C.spectabilis et le témoin, respectivement (Figures $1 \mathrm{c}$ et $1 \mathrm{~d}$ ). La hauteur des plants bisannuels de $T$. vogelii et $S$ sesban avait diminué de $200 \mathrm{~cm}$ à $164,8 \mathrm{~cm}$ et à $147,4 \mathrm{~cm}$ respectivement comparable au témoin $152,5 \mathrm{~cm}$ en troisième saison. Pendant que les rendements décroissants de maïs de T.vogelii à $1470,4 \mathrm{~kg} / \mathrm{ha}$ et de $S$. sesban à $1615,2 \mathrm{~kg} / \mathrm{ha}$ face au témoin décroissant à $1164 \mathrm{~kg} / \mathrm{ha}$ avaient aussi été significativement inférieurs à ceux de L.leucocephala et C.spectabilis avec respectivement $3088,8 \mathrm{~kg} / \mathrm{ha}$ et $2648,8 \mathrm{~kg} / \mathrm{ha}$. Des rendements du maïs relativement plus élevés sous $T$. vogelii et $S$. sesban pourraient être liés à des teneurs plus élevées en éléments minéraux nutritifs mobiles : $\mathrm{N}, \mathrm{Ca}, \mathrm{K}$ et $\mathrm{Na}$ [Kang et al., 1997; Tian et al., 1993] relatives aux quantités de leurs biomasses. De même, les plus faibles rendements sous C.spectabilis et L.leucocephala par rapport au témoin seraient probablement dus à la nature tardive de leur 
végétation et/ou à l'activité nodulaire moins intense de $L$. leucocephala par rapport aux $T$. vogellii et $S$. sesban (tableau 2).

\section{L'effet d'applications continues des} biomasses des arbustes pérennes sur le maïs : Les arbustes bisannuels ayant dépéris, l'effet d'application continue des biomasses de $L$. leucocephala et C. spectabilis (figure 1a) en deuxième et troisième saisons culturales a affecté significativement la croissance et le rendement du maïs (figures $1 \mathrm{~b}, 1 \mathrm{c}$ et $1 \mathrm{~d}$ ). Plusieurs tendances peuvent être distinguées :

+ 1. Pendant qu'en troisième saison, le diamètre au collet du maïs traité à $L$. leucocephala a diminué de 2,01 à $1,74 \mathrm{~cm}$ mais significativement supérieur à celui du témoin diminué à $1,36 \mathrm{~cm}$; le diamètre de maïs sous $C$. spectabilis a très peu varié, soit de $2,11 \mathrm{~cm}$ à $1,96 \mathrm{~cm}$ (figure $1 \mathrm{~b}$ ).

$+\quad 2$. La hauteur du maïs sous $L$. leucocephala (de $185 \mathrm{~cm}$ à 216,6 cm) en troisième saison (figure $1 \mathrm{c}$ ) et sous $C$. spectabilis $(221,9 \mathrm{~cm})$ en deuxième saison a significativement augmenté par rapport au témoin dont la hauteur la plus faible est observée à la troisième saison (figure 1c).

+ 3. Les grandes biomasses de $L$. leucocephala (figure 1a) produites constamment en deuxième $(18,15 \mathrm{t} / \mathrm{ha})$ et troisième $(17,88$ t/ha) saisons ont triplé les quantités minérales de $\mathrm{P}, \mathrm{K}, \mathrm{Ca}$ et $\mathrm{Mg}$ (tableau 3) dont les plus élevées étaient Ca $101.5 \mathrm{~kg} / \mathrm{ha}$ et $\mathrm{K} 31,8 \mathrm{~kg} / \mathrm{ha}$ avec $\mathrm{Mg}$ et $\mathrm{P}$ inférieures à $8 \mathrm{~kg} / \mathrm{ha}$. Ces phyto-masses auraient amélioré, outre la nutrition minérale du maïs et de L. leucocephala, l'activité rhizobiale spécifique de $L$. leucocephala dans la fixation d'azote et accéléré le développement du maïs (Kombele, 2004 ; Van Laure et al., 1999 ; Kang et al., 1991). Cet apport minéral par $L$. leucocephala aurait ainsi stabilisé la production du maïs à 2998,8 et $3088,8 \mathrm{~kg} / \mathrm{ha}$ au cours de deux dernières saisons culturales.

+ 4. Ces rendements du maïs sous $L$. leucocephala sans engrais de fond n'est pas aussi loin des résultats de Kang et al. (1997) qui observaient en sept ans un rendement de 3,5 $t / h a$ et $4,5 t / h a$ de maïs respectivement sans et avec engrais minéral, et chaulage avec les biomasses de $L$. leucocephala sur des alfisols. Aussi, Sanginga et al. (1995) récoltaient en dix ans 4,2 t/ha de maïs par un inoculum fixateur d'azote et un engrais de fonds avec les biomasses de L. leucocephala. Sur des ferralsols de Kisangani (à $100 \mathrm{~km}$ de Yangambi en RD Congo), 22,0 tha de phytomasses de $L$. leucocephala en culture en couloirs produisaient 3,0 t/ha de mails et 16,0 tha de bananes plantains (Mate, 2003). Concernant le C. spectabilis, le deuxième arbuste bisannuel sous étude, au cours de la deuxième saison culturale, la hauteur $(221,9 \mathrm{~cm}$, figure $1 \mathrm{c})$ et le rendement du maïs $(3444,8 \mathrm{~kg} / \mathrm{ha}$, figure $1 \mathrm{~d})$ ont été plus élevés par rapport à la première saison. Cette tendance résulterait de la grande quantité de biomasse foliaire $(22,98 \mathrm{t} / \mathrm{ha}$ ) ayant produit six fois la quantité de $\mathrm{K}$ ( $60 \mathrm{~kg} / \mathrm{ha}$ ), trois fois celle de Ca $(108,8 \mathrm{~kg} / \mathrm{ha})$ et quadruplant celles de $\mathrm{P}$ $(11,6 \mathrm{~kg} / \mathrm{ha})$ et $\mathrm{Mg} 11,9 \mathrm{~kg} / \mathrm{ha})$. Ceci serait suite à l'excellent pouvoir rejet tonnant de souches de L. leucocephala accompagné de formation rapide du massif végétal pendant 9 mois de jachérisation en période de la grande saison humide. En troisième saison culturale, par contre, la baisse du rendement du maïs de $29 \%$ par rapport à la deuxième saison avec $C$. spectabilis, correspondait à la faible quantité de biomasse de 1,02 tha qui serait due à sa nature non-nodulaire, au manque d'engrais de fonds et à une courte jachérisation de 3 mois de juillet à septembre.

Moyennes globales de trois saisons culturales successives: Les résultats moyens globaux des légumineuses pérennes en trois saisons culturales successives sur le maïs présentés au tableau 3 montrent que les différences significatives entre les moyennes globales des paramètres de maïs seraient causées par les quantités élevées de phytobiomasses cumulées de L.leucocephala $(42,11$ t/ha) et C.spectabilis (36,38 t/ha) ayant généré proportionnellement la fertilité du sol par les minéraux et par l'absence de T.vogelii et S.sesban en deuxième et troisième saisons. Cette absence des bisannuels avaient présenté des faibles valeurs moyennes significatives respectivement de $1,64 \mathrm{~cm}$ et $1,67 \mathrm{~cm}$ de diamètre, $171,53 \mathrm{~cm}$ et $181,14 \mathrm{~cm}$ de hauteur et $1986,27 \mathrm{~kg} / \mathrm{ha}$ et $2092,13 \mathrm{~kg} / \mathrm{ha}$ de rendement de maïs par rapport aux pérennes. Les hauteurs moyennes de pérennes allant jusqu'à $206 \mathrm{~cm}$ et leurs rendements respectifs de 2759,20 et $2694,53 \mathrm{~kg} / \mathrm{ha}$ avaient été significativement supérieurs. Plus de $85 \%$ de N, P et $\mathrm{Ca}$ des biomasses incorporées seraient adsorbés sur le complexe d'échange cationique du sol. Le pH s'est élevé de 4,88 à 5,71 et 5,54 respectivement pour les sols à $L$. leucocephala et $C$. spectabilis. L'évolution positive en hauteur et rendement du maïs et en phytomasses des pérennes démontrerait que les rapports $(\mathrm{Ca}+\mathrm{Mg}) / \mathrm{K}$, 
$\mathrm{Ca} / \mathrm{Mg}$ et $\mathrm{C} / \mathrm{N}$ auraient été satisfaisants dans les sols (Alongo et al., 2013; Van Laure et al., 2000 ; Kang et 1997).

L'arrière effet des biomasses des arbustes bisannuels sur le maïs :L'arrière effet des biomasses de T.vogelii et S.sesban dépéris après deux tailles de la première compagne culturale (figure 1a) sur la réponse du maïs en

\section{CONCLUSION}

L'influence des biomasses de quatre légumineuses ligneuses a été évaluée sur le rendement de maïs variété KASAÏ 1 semée sans engrais de fonds sur le ferralsol de série $Y_{2}$ de Yangambi. En trois saisons culturales successives, il s'est avéré qu'en première saison, les arbustes précoces bisannuels Tephrosia vogelii et Sesbania sesban ont produit des quantités élevées de biomasses relatives aux rendements de maïs par rapport au témoin respectivement de $+8,41 \%$ (2726 $\mathrm{kg} / \mathrm{ha})$ et $+13,2 \% \quad$ (2846 kg/ha) qui n'ont pas été significativement différent de ceux des arbustes pérennes Leucaena leucocephala -11,5\% (2220 $\mathrm{kg} / \mathrm{ha}$ ) et Cassia spectabilis -20,0\% (1991 kg/ha). En deuxième et troisième saisons, l'arrière-effet des arbustes bisannuels dépéris a montré des valeurs décroissantes des paramètres observés comparables au témoin. Par contre à cause des quantités élevées des biomasses de légumineuses pérennes, proportionnelles aux éléments minéraux majeurs produits, $L$. leucocephala a augmenté le rendement du maïs de $+11,66 \% \quad(2968,8 \mathrm{~kg} / \mathrm{ha})$ et $+164,23 \%$ $(3088,8 \mathrm{~kg} / \mathrm{ha}$ ) et le C. spectabilis de $+29,60 \%$ $(3444,8 \mathrm{~kg} / \mathrm{ha})$ et de $+126,59 \%(2648,8 \mathrm{~kg} / \mathrm{ha})$ par rapport au témoin.En moyennes de trois saisons culturales, les biomasses de $L$. leucocephala et de C. spectabilis ont

\section{REFERENCES BIBLIOGRAPHIQUES}

Alongo Sylvain, Marjolein Visser, Ferdinand Kombele, Gilles Colinet, Jean Bogaert, 2013. Propriétés et diagnostic de l'état agropédologique du sol de la série Yakonde après fragmentation de la forêt à Yangambi, R D CONGO. Annales des instituts supérieurs d'études agronomiques, 2013, 5(1), pp36-51. <hal-00875748>

Alongo, S., MBELI, P., Kombele, F., 2014. Agriculture itinérante sur brûlis à Yangambi (RDC) : vers une transition agraire dans le contexte de conservation des forêts et sécurité couloir a été nul en deuxième et troisième saisons. Car les faibles valeurs décroissantes de hauteur, diamètre et rendement observées en figures $1 \mathrm{~b}, 1 \mathrm{c}$ et $1 \mathrm{~d}$ avaient été comparables au témoin et significativement inférieures aux valeurs croissantes de ces paramètres de maïs sous L.leucocephala et C.spectabilis.

significativement influencé la croissance du diamètre moyen du maïs à 1,83 et $1,99 \mathrm{~cm}$, les hauteurs moyennes à $206 \mathrm{~cm}$ et les rendements moyens de 2759,2 et 2694,5 kg/ha. La nature de la courte jachérisation herbeuse entre les saisons, associée à ces légumineuses ligneuses pourrait aussi jouer un rôle additionnel dans la minéralisation des éléments chimiques majeurs du sol. L'espèce locale $C$. spectabilis serait préférable par rapport au $L$. leucocephala plus productive qui n'a pas été dans son écologie d'alfisol savanicole. La replantation des bisannuels en chaque saison demanderait une main d'œuvre supplémentaire. L'obtention d'engrais de fonds n'étant pas facile pour les agriculteurs, l'effort peut être déployée pour identifier et utiliser :

-les inocula fixateurs d'azote adaptés aux légumineuses ligneuses et herbeuses sur des ferralsols de Yangambi pour augmenter et stabiliser les rendements des cultures;

-les légumineuses ligneuses et herbeuses locales dans la biodiversité floristique de la réserve de Yangambi

-l'efficience se rapportant à la quantité des biomasses d'un arbuste à l'unité de surface de terre cultivée pour élever le rendement de maîs à l'optimum devra être étudié.

alimentaire. Poster, Institut Facultaire des Sciences Agronomiques de Yangambi, laboratoire d'agroécologie, pédopaysage et système agraire, B.P. 1232 Kisangani, R.D. Congo

FAO (Food and Agriculture organization), 2000. Politiques agricoles pour la gestion et l'utilisation durables des ressources naturelles en Afrique. IITA, Ibadan, livre 96p.

Kang B.T., Gichuru, N. Hulugalle, et Swift, M.J., 1991. Soil constraints for sustainable upland crop production in humid and subhumid West-Africa. 76p. 
Kang, B.T., Salako, A.F.K., Akobundu, I.O., Pleysier, J.L. and Chiann, J.N., 1997. Amelioration of a degraded oxic paleustalf by leguminous and natural fallows. Soil use and management $13: 130-136$.

Kombele, B., 2004. Diagnostic de la fertilité des sols dans la Cuvette Centrale Congolaise. Cas des séries Yangambi et Yakonde. Thèse doctorale en sciences agronomiques, faculté universitaire de Gembloux, Belgique. 464p.

Jurion, F. et Henri, J., 1967. De l'agriculture itinérante à l'agriculture intensive. Publication INEAC, hors-série, 487p.

INERA (Institut National pour l'Etude et la Recherche Agronomiques), 2010. Rapport annuel 2010, Centre de Yangambi.

Likoko, B. et Tentula K., 2003. Adventices dans la riziculture pluviale sur quelques oxisols de Yangambi. Annales de la faculté des sciences, UNIKIS. Pp 449457.

Likoko, B., Alongo, L. et Likoko, A.G., 2014. Perturbations de tendances climatiques actuelles de la région de Yangambi. Abstract $\mathrm{N}^{\circ} \mathrm{C} 143$, Première conférence internationale sur la biodiversité dans le bassin du Congo. Pp 135.

Letouzey et White F., 1978. La flore du Gabon.

Mate J.P.M., 2003, Croissance, phytomasse et minéralomasse des haies des légumineuses améliorantes en cultures en allées à Kisangani. Thèse doctorale, Gembloux, Belgique

Sanginga, N., Danso, S.K.A., Mulongoy, K. et Ojeifo, A.A., 1995. Persistance et reprise de rhizobium introduit dix ans après inoculation sur Leucaena cultivé sur un alfisol dans le sud-ouest du Nigeria. La recherche à I'ITTA, marsseptembre 1995.

Tian, G., Kang, B.T. and Brussard, L., 1994. Effet du paillage de débris végétaux à compositions chimiques contrôlées sur la croissance du maïs et l'accumulation des éléments nutritifs. La recherche à I'IITA, No 9, pp 7-11.

Landon J.R., 1991. Booker tropical soil manual. A handbook for soil survey and agricultural land evaluation in the tropics and subtropics. Oxon, UK: Booker Tate Limited; Harlow, Essex, UK: Longman.

Van Laure, B., Aihou, K., Iwuafor, E.N.O., Hougnandan, P., Diels, J. Manyong V.M.

and Sanginga, N., 2000. Recent development in soil fertility management of maize-base systems; the role of legumes in $\mathrm{N}$ and $P$ nutrition of maize in the moist savana zone of West

Africa. Pp 3-13. Proceedings of workshop held in Cotonou, Benin 26-29 october 1999. In cover crop for natural resource management in West 1 Africa 